\title{
Numerical Study on Self-Cleaning Canister Filter With Add-On Filter Cap
}

\author{
Akmal Nizam Mohammed ${ }^{1,1}$, Mohd Sahrizan Sahrudin ${ }^{1}$, Mohd Hafiz Zolkhaely ${ }^{1}$, Azwan \\ Sapit ${ }^{1}$, Mohd Kamil Abdullah ${ }^{1}$, Suzairin Md Seri ${ }^{1}$, Ishkrizat Taib ${ }^{1}$ and Normayati Nordin ${ }^{2}$ \\ ${ }^{1}$ Faculty of Mechanical and Manufacturing Engineering, Universiti Tun Hussein Onn Malaysia \\ ${ }^{2}$ Flow Analysis, Simulation, and Turbulence Research Group, Universiti Tun Hussein Onn Malaysia
}

\begin{abstract}
Filtration in a turbo machinery system such as a gas turbine will ensure that the air entering the inlet is free from contaminants that could bring damage to the main system. Self-cleaning filter systems for gas turbines are designed for continuously efficient flow filtration. A good filter would be able to maintain its effectiveness over a longer time period, prolonging the duration between filter replacements and providing lower pressure drop over its operating lifetime. With this goal in mind, the current study is focused on the difference in pressure loss of the benchmark Salutary Avenue Self-cleaning filter in comparison to a new design with an add-on filter cap. Geometry for the add-on filter cap will be based from Salutary Avenue Manufacturing Sdn.Bhd. SOLIDWORKS software was used to model the geometry of the filter, while simulation analysis on the flow through the filter was done using Computational Fluid Dynamic (CFD) software. The simulations are based on a low velocity condition, in which the parameter for the inlet velocity are set at $0.032 \mathrm{~m} / \mathrm{s}, 0.063 \mathrm{~m} / \mathrm{s}$, $0.094 \mathrm{~m} / \mathrm{s}$ and $0.126 \mathrm{~m} / \mathrm{s}$ respectively. From the simulation data obtained for the inlet velocities considered, the pressure drop reduction of the modified filter compared to the benchmark was found to be between $7.59 \%$ and $30.18 \%$. All in all, the modification of the filter cap produced a lower pressure drop in comparison with the benchmark filter; an improvement of $27.02 \%$ for the total pressure drop was obtained.
\end{abstract}

\section{Introduction}

Filtration is one of the essential parts in a gas turbine. Filters are used to prevent foreign contaminants in air from entering the system in a gas turbine. Air is drawn into the turbine by using compressors until it reaches the combustion chamber, in which fuel is sprayed into the air and ignited to generate a high-temperature flow. In a gas turbine, its efficiency is its most important characteristic, and the factors that can affect its efficiency are many and various. Among the main factors that will affect the efficiency of a gas turbine is thru its

${ }^{1}$ Corresponding author: akmaln@uthm.edu.my. 
inlet. As the gas turbine cycle operates, both its inlet and exhaust are open to the atmosphere, and hence the flow parameters would vary with atmospheric conditions. Contaminants in the air that consist of different types and from a variety of environments can cause several kinds of problems which can negatively impact the reliability, availability, and time between overhauls of gas turbine internal components thus affecting its efficiency.

The efficiency of the gas turbine can also be affected by the air inlet temperature of the gas turbine. Based from study of [1] among the method to increase the performance and efficiency of a gas turbine is by air cooling and filtration system in the inlet of a gas turbine. It is well known that ambient temperature, humidity and pressure are important factors in gas turbine performance. As mentioned in [2], thermodynamic analyses exposed that thermal efficiency and specific output decrease with an increase of humidity and ambient temperature.

The selection of the filtration system must be taken into consideration as there are many factors need to be considered in choosing filtration system in a gas turbine. As mentioned in [3] the system of filtration for a gas turbine are selected based on the operational philosophy and goals for the turbine, the contaminants that are present in the ambient air, and also based on expected changes in the contaminants in the future due to temporary emission sources or seasonal changes.

Increase in the pressure drop will causes an increase in energy consumption. Based from study of [4] Pressure loss indicates the resistance of airflow through a filter. High filter pressure drop reduces turbine output and a low pressure drops improves turbine output. To overcome the inlet system losses, the gas turbine will consume more fuel, and it also has to reduce its power output. The pressure loss will increases, while the power will decreases and the heat rate will increases linearly.

Self-cleaning filters are used in low approach velocity systems thus when normal flow is re-established the low air velocity assists in preventing dust that is being removed from the filter with air pulsing from re-entering again in the airflow stream. As the surface of the filter is loaded with particles, it decreases the available flow area through the filter which increases their efficiency. This type of filtration system is very useful in dry environments with high levels of dust. While in high moisture environments the selfcleaning filter is prone to high pressure losses and other problems.

Computational Fluid Dynamic (CFD) techniques have the potential to allow the effect of a proposed design change to be evaluated relatively quickly while the cost can be keep at a minimum rather than doing experimental investigation. As mentioned in [5], detailed information such as velocity, pressure, temperature, concentration and, turbulence intensity can also be obtain by using this method. The study aims to give further understanding about suitable design of a gas turbine canister filter so that an increase in gas turbine efficiency can be achieved. The analysis of the pressure drop in the gas turbine canister filter are made using CFD software so that the cost of producing prototype for the canister filter can be reduced.

\section{Methodology}

The simulation and numerical analysis method of the whole self-cleaning canister filter are made using Computational Fluid Dynamics software that is the Workbench16.1. In CFD, the process for the numerical analysis is divided into three stages which are pre-processing, solvers and post-processing. The type of analysis conducted using this software is Fluid Flow (CFX).

The parameter for the simulations are based from Salutary Avenue (SA) brochure, the data that are available from Salutary Avenue (SA) are the air flow rate, surface area of 
the filter and also the pressure drop for the experiment of Salutary Avenue (SA) filter. The data that are obtained from Salutary Avenue (SA) are for the F9 type filter. From the data that available, Table 1 are build based from the data.

Table 1. Parameter for simulations of benchmark and modified filter.

\begin{tabular}{|c|c|c|c|}
\hline Flowrate $\left(\mathbf{m}^{3} / \mathbf{s}\right)$ & Pressure Drop $(\mathbf{P a})$ & Area $\left(\mathbf{m}^{2}\right)$ & Velocity $(\mathbf{m} / \mathbf{s})$ \\
\hline 0.236 & 25 & 7.5 & 0.032 \\
\hline 0.472 & 47.23 & 7.5 & 0.063 \\
\hline 0.708 & 77.1 & 7.5 & 0.094 \\
\hline 0.944 & 121.81 & 7.5 & 0.126 \\
\hline
\end{tabular}

Pre-processing is where the geometry of the item is determine than is drawn either using ANSYS Workbench or SOLIDWORKS software. For this study the geometry of the selfcleaning canister filter with add on filter cap is drawn by using SOLIDWORKS it is then generated into the Design Modeller (DM) of ANSYS workbench as showed in Figure-1 (a). Modelled of the geometry is then need to be computational mesh throughout the flow volume as in Figure-1 (b).
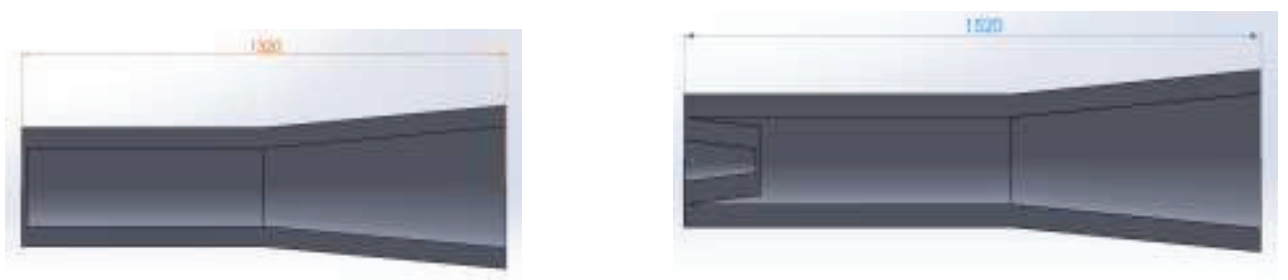

(b)

Fig. 1. Benchmark filter of original size is shown in (a), whilst the modified model is given in (b).

Mesh is the well organize arrangement of flow field at discrete point in a flow domain. Long solver runs may due to too many cells, while inaccurate results may cause by too few of cells. The last step in pre-processing is setup. In this step, parameters for the model need to be declared. Parameters such as domain, boundary condition and the medium of the material need to be specifically declared so that the setup process and solution solver of the model can be obtain. The model are split into two domain that is air domain and filter domain.

Solver is an unstructured grid finite volume solver for the compressible flow where the previous inputted data from the pre-processing step are calculated. The solver setting will calculate based on the number of iteration for the model geometry. The number of iteration can be set on the solver setting; once the number of target residuals are achieved, the solver will stop even though the number of maximum iterations are not achieved, the solver is converge at 0.0001 residual target with the number of iterations is generated on the output file.

The last step in ANSYS Fluent software is the post-processing where the simulation of the model are generated, calculated data in the solver is transferred into figures, meaningful graphics, animations and reports that make it easy to convey the fluid dynamic results. For the post-processing result, a line will generated based from the length dimensions for the filter, the data will be extracted and export that are based from the line that is generated. 
Grid independency test is done to identify the best meshing size by saving the computation time without neglecting the accuracy of the results, meshing is a break-down of a single unit geometry into a small discrete control volume where the set of equations are applied on each cell and computed.. The grid independency test is done over a range of grid size to compare and obtain the best and minimum computational time. Table- 2 show the different grid sizes with its number elements and nodes. The medium nodes of 337697 were selected as the results is not far apart to the higher nodes of 445034 and minimum computational time will occurred

Table 2. Grid size setting for coarse, medium, and fine meshes respectively.

\begin{tabular}{|c|c|c|c|}
\hline \multicolumn{2}{|c|}{ Grid Size Setting } & \multirow{2}{*}{ No of Nodes } & No of Element \\
\cline { 1 - 2 } Min & Max & & \\
\hline 8 & 13 & 207677 & 1118435 \\
\hline 8 & 11 & 337697 & 1845530 \\
\hline 7 & 10 & 445034 & 2451446 \\
\hline
\end{tabular}

\section{Results and Discussion}

The pressure drop is calculated by subtracting the outlet pressure from the inlet pressure, as shown in Eqn. (1). The value of the pressure drop are calculated by getting the pressure value by assuming the inlet pressure is at the narrow end of the filter minus the outlet pressure at wider end.

$$
\Delta P=P_{\text {inlet }}-P_{\text {outlet }}
$$

Three dimensional velocity streamline for the benchmark and modified filter simulation can give a clear view of the air flow that enter the filter as showed in Figure 2, from the figure, it showed that the air that enter the filter is from the front and surrounding of the filter, by modifying the filter, a better flow of air is seen entering the filter, air can enter from the front for the modified filter rather than the benchmark filter, where wall is placed in front of the filter.

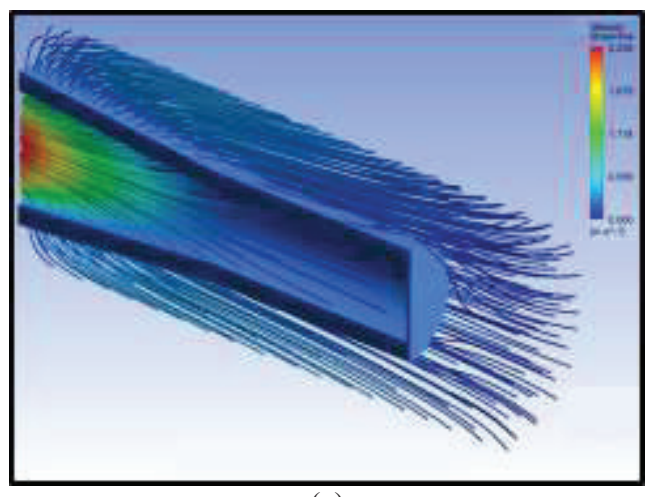

(a)

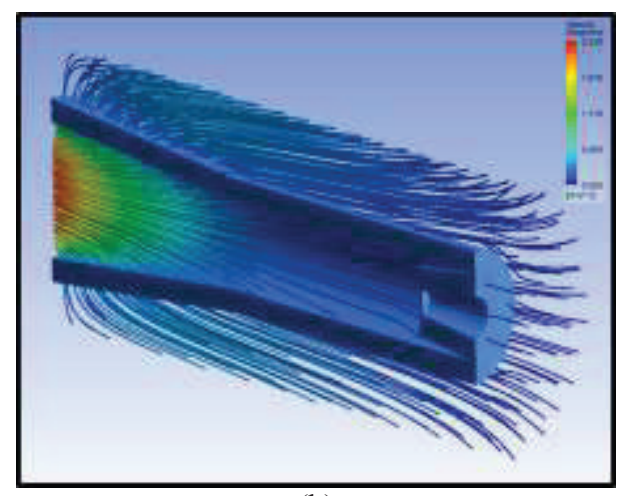

(b)

Fig. 2. Benchmark filter of original size is shown in (a), whilst the modified model is given in (b). 
The simulation of benchmark filter, showed a lower pressure drop compared to the Salutary Avenue (SA) at lower inlet velocity of $0.032 \mathrm{~m} / \mathrm{s}$ and $0.063 \mathrm{~m} / \mathrm{s}$, while at the last two velocity inlet parameter of $0.094 \mathrm{~m} / \mathrm{s}$ and $0.126 \mathrm{~m} / \mathrm{s}$ it showed that the Salutary Avenue (SA) has a lower pressure drop than simulation of benchmark filter as show in Figure 3a. The value of the result vary because the experimental data for Salutary Avenue (SA) is not fully revealed, thus leaving assumption need to be made at the boundary condition for simulation of the benchmark filter.

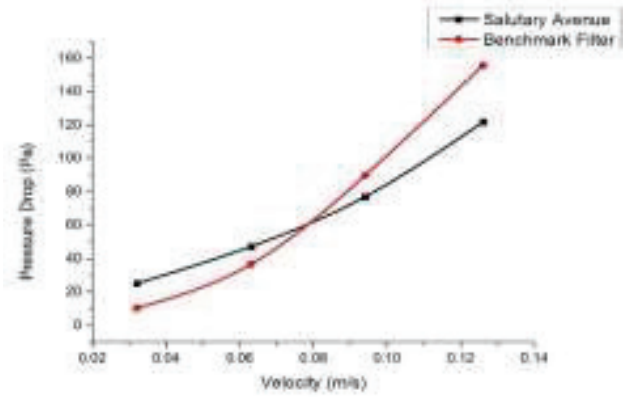

(a)

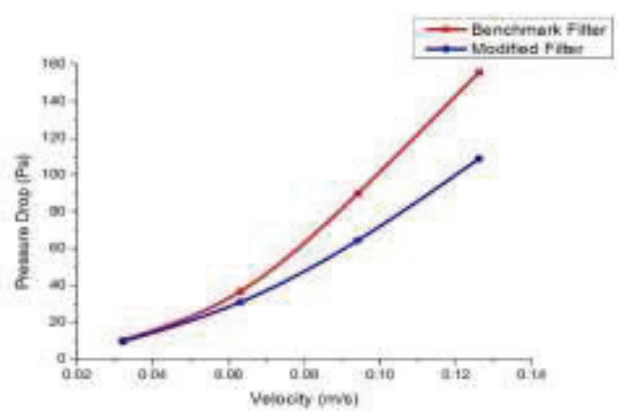

(b)

Fig. 3. Benchmark filter of original size is shown in (a), whilst the modified model is given in (b).

For comparison of simulation for the benchmark and modified filter, it is more likely because all of the simulation setting are the same for the simulation except for the geometry of the filter, it showed that the pressure drop for the modified filter is better than the modified filter, the pressure drop for inlet velocity of $0.032 \mathrm{~m} / \mathrm{s}$ is not far apart as benchmark filter recorded 10.191 Pascal of pressure drop while 9.418 Pascal for the modified filter, as the inlet velocity parameter became larger, the pressure drop increased with an increase of velocity.

Lastly, a comparison of all the data had been made for Salutary Avenue (SA) based from the graph on Figure $3 \mathrm{~b}$ and Table 3 for benchmark and modified filter. Based from the plotted graph, it showed that the modified filter had a lower pressure drop compared with Salutary Avenue (SA) and the benchmark filter, the modified filter had a better pressure drop due to the additional area for the modified filter. The modified cap of the filter, enable air to flow from the front area of the filter, compared than benchmark filter where wall is place in front of the filter, restricted the airflow entering the filter. The additional surface area for the modified filter made the filter had a lower pressure drop than others.

Table 3. Tabulated values of pressure drop for the simulated filter compared with benchmarks.

\begin{tabular}{|c|c|c|c|}
\hline \multirow{2}{*}{ Velocity (m/s) } & \multicolumn{3}{|c|}{ Pressure Drop (Pa) } \\
\cline { 2 - 4 } & Salutary Avenue & Benchmark Filter & Modified Filter \\
\hline 0.032 & 25.00 & 10.19 & 9.42 \\
\hline 0.063 & 47.23 & 36.63 & 30.85 \\
\hline 0.094 & 77.10 & 89.88 & 64.44 \\
\hline 0.126 & 121.81 & 155.77 & 108.75 \\
\hline
\end{tabular}




\section{Conclusion}

It can be concluded that the objective of the research had been achieved. The cases are based on a low velocity system for velocity inlet parameter of $0.032,0.063,0.094$ and $0.126 \mathrm{~m} / \mathrm{s}$. The parameter for obtaining a low pressure drop in a Salutary Avenue filter had been recognised, with an increase of its overall filter length of $20 \mathrm{~cm}$, a total pressure drop of 213.456 Pascal can be achieved for simulation of modified filter, the result is $27.02 \%$ better than the simulation result from total pressure drop of benchmark filter that are 292.469 Pascal for total set parameter of inlet velocity. The range for pressure drop is between $7.59 \%$ and $30.18 \%$ for inlet velocity parameter range of 0.032 until $0.126 \mathrm{~m} / \mathrm{s}$ for simulations comparison of benchmark and modified filter. The pressure drop can be lower if the area of the filter are increased, in this case is by increasing the overall filter length. The pressure drop for the filter is not only determined on the area of the filter, it also are due to the design of the inlet system ducting also, due to lack of experimental results and parameters from Salutary Avenue, the data that are obtain from simulation may not be highly precise, this is because the data that are not fully reveal for this study, and limited information that are able to be obtained.

\section{Acknowledgement}

This research was made possible through funding from UTHM IGSP Grant U413 and UTHM STG Grant U126. The authors would also like to acknowledge the contributions made by members of Flow Analysis, Simulation and Turbulence Research Group (FAST), The Centre for Energy and Industrial Environment Studies (CEIES) of UTHM, and Salutary Avenue Manufacturing Services Sdn. Bhd.

\section{References}

1. A. $\mathrm{Ku}$, Comparative analysis of inlet air cooling techniques integrated to cooled gas turbine plant. Journal of the Energy Institute, 1-15 (2014).

2. M. Farzaneh-Gord, M. Deymi-Dashtebayaz, Effect of various inlet air cooling methods on gas turbine performance. Energy, 36(2), 1196-1205 (2011).

3. M. Wilcox, R. Kurz, K. Brun, Technology review of modern gas turbine inlet filtration systems. International Journal of Rotating Machinery (2012).

4. Z. Feng, Z. Long, Q. Chen, Assessment of various CFD models for predicting airflow and pressure drop through pleated filter system. Building and Environment, 75, 132141 (2014).

5. C. Tsang, Analysis of pleated air filters using computational fluid dynamics. University of Toronto (1997). 